\title{
Beam-loading effect on breakdown rate in high-gradient accelerating cavities: An experiment at the Compact Linear Collider Test Facility at CERN
}

\author{
Eugenio Senes, ${ }^{1,2, *}$ Theodoros Argyropoulos, ${ }^{3,2}$ Frank Tecker, ${ }^{2}$ and Walter Wuensch ${ }^{2}$ \\ ${ }^{1}$ John Adams Institute and University of Oxford, The Denys Wilkinson Building, \\ Keble Road, Oxford OX1 3RH, United Kingdom \\ ${ }^{2}$ CERN, 1211 Geneve 23, Switzerland \\ ${ }^{3}$ IFIC (CSIC-UV), 46100 Valencia, Spain
}

(Received 16 February 2018; published 19 October 2018)

\begin{abstract}
Radio frequency breakdown rate is a crucial performance parameter that ensures that the design luminosity is achieved in the CLIC linear collider. The required low breakdown rate for CLIC, of the order of $10^{-7}$ breakdown pulse $\mathrm{e}^{-1} \mathrm{~m}^{-1}$, has been demonstrated in a number of $12 \mathrm{GHz}$ CLIC prototype structures at gradients in excess of the design $100 \mathrm{MV} / \mathrm{m}$ accelerating gradient, however without the presence of the accelerated beam and associated beam loading. The beam loading induced by the approximately $1 \mathrm{~A}$ CLIC main beam significantly modifies the field distribution inside the structures, and the effect on breakdown rate is potentially significant so needs to be determined. A dedicated experiment has been carried out in the CLIC Test Facility CTF3 to measure this effect, and the results are presented.
\end{abstract}

DOI: 10.1103/PhysRevAccelBeams.21.102001

\section{INTRODUCTION}

The CLIC [1] TeV-range linear collider aims to collide electrons and positrons accelerated in two opposing linacs using normal-conducting high-gradient accelerating structures. A major limitation to the achievable gradient are $\mathrm{rf}$ breakdowns (BD), which inhibit power propagation and induce transverse kicks to the beam causing luminosity loss [2]. The specification for maximum breakdown rate (BDR) is $3 \times 10^{-7} \mathrm{BD} /($ pulse $\cdot \mathrm{m}$ ) for the $3 \mathrm{TeV}$ CLIC, chosen in order to limit luminosity loss due to breakdowns to less than $1 \%$. An intensive high-gradient testing program has been carried out, and results demonstrate that such low breakdown rates are achievable in excess of the $3 \mathrm{TeV}$ CLIC design gradient of $100 \mathrm{MV} / \mathrm{m}[3,4]$. These tests have been carried out without beam inside the structure.

The beam presence produces additional fields inside the accelerating structure, that combined with the injected $\mathrm{rf}$ modify the internal field distribution. The field modification is known as beam loading [5,6]. CLIC is designed for a high rf-to-beam efficiency requiring a high level of beam loading. This is accomplished with a high beam current of approximately $1.2 \mathrm{~A}$, unavoidably modifying the longitudinal field profile. The input power to the structure needs

\footnotetext{
*eugenio.senes@cern.ch
}

Published by the American Physical Society under the terms of the Creative Commons Attribution 4.0 International license. Further distribution of this work must maintain attribution to the author(s) and the published article's title, journal citation, and DOI. to be increased accordingly to maintain the same average accelerating gradient inside the structure.

The effect of modifying the internal field profile on the BDR is hard to predict since two very different dependencies are observed during experiments with varying electric field profiles [7]. When the input power to a structure is varied, the whole-structure BDR varies approximately as

$$
\mathrm{BDR} \propto E_{\mathrm{acc}}^{30}
$$

where $E_{\text {acc }}$ is the accelerating field [7]. On the other hand, the distribution of the longitudinal position of the breakdowns indicates a linear proportionality to the peak surface electric field [8]. The dynamic change of breakdown rate when the field profile is changed by introducing beam is expected to lie somewhere between these two dependencies.

In order to directly experimentally measure the effect of beam loading on BDR, an experiment has been carried out [9] in the CLIC Test Facility (CTF3) at CERN [10]. The rf power is provided by a $12 \mathrm{GHz}$ klystron and the beam is taken from the electron linac of the facility. Initial measurements with the beam can be found in $[11,12]$, and this work presents a significantly improved set of measurements also with better statistics.

\section{EXPERIMENT LAYOUT}

The CTF3 Drive Beam linac [13] can be operated to produce a CLIC Main-Beam-like electron beam with the parameters reported in Table I. Branching halfway off the 
TABLE I. Beam parameters used for the experiment and CLIC $3 \mathrm{TeV}$ design parameters $[1,14]$.

\begin{tabular}{lcc}
\hline \hline & This experiment & CLIC 3 TeV \\
\hline Beam current [A] & 1.6 & 1.2 \\
Pulse length [ns] & 330 & 156 \\
Energy [GeV] & 0.13 & 9 to 3000 \\
Repetition rate [Hz] & 25 & 50 \\
Bunch charge [pC] & 530 & 600 \\
Bunch separation [ns] & 0.33 & 0.5 \\
Number of bunches & 984 & 312 \\
$\epsilon_{x, \text { normalised }}$ & $75-105 \mu \mathrm{m}$ & $660 \mathrm{~nm}$ \\
$\epsilon_{y, \text { normalised }}$ & $70-130 \mu \mathrm{m}$ & $20 \mathrm{~nm}$ \\
Relative energy spread & $2 \%$ & $<2 \%$ to $0.35 \%$ \\
\hline \hline
\end{tabular}

linac, a parallel beamline (called dogleg due to the shape) has been used for this experiment.

The beamline optics has been designed to provide minimum size in and maximum transmission through the structure under test [11]. An $8 \mathrm{~mm}$ aperture collimator is installed upstream the structure to protect it from beam losses. Two beam position monitors are installed one upstream and one downstream the structure. The beam properties can be measured by sending it down the linac instead of the dogleg line. A spectrometer measures energy, and a screen is used to measure energy and Twiss parameters of the beam by a quadrupole scan.

The structure under test is fed with high power rf field by a $12 \mathrm{GHz}$ source $[15,16]$. The basic layout of the experimental setup is shown in Fig. 1. The rf power generation starts with the low level rf signal (LLRF) that is preamplified by a travelling wave tube (TWT) and then propagated in the XL-5 klystron [17]. The klystron is capable of producing a $50 \mathrm{MW}$ rf power pulse at $12 \mathrm{GHz}$ of $1.5 \mu$ s length at $50 \mathrm{~Hz}$ repetition rate driven by a Scandinova solid state modulator [18]. The klystron output pulse is compressed by a SLED-I [19] pulse compressor into a $250 \mathrm{~ns} 140 \mathrm{MW}$ pulse [20]. The waveguide network of around $35 \mathrm{~m}$ length has a power transmission coefficient of $67 \%$ with 115 ns group delay time.

The forward, reflected and transmitted rf power are measured through directional couplers before and after the structure. These signals are acquired by logarithmic detectors. I/Q demodulators are also installed on the three acquisition channels and provide information on the phase of the signals. The two beam position monitors (BPMs) upand downstream the structure measure the beam current and transmission.

A National Instruments PXIe-8133 controller equipped with high-performance NI FlexRIO FPGA-based digitisers (NI5761-250 MSa/s-4ch) [21] acquires signals and interlocks the system. It is also complemented with serial buses, digital IO and links to the CERN control system, all controlled and interfaced to the user with an adapted version of the Labview program used in the test stand.

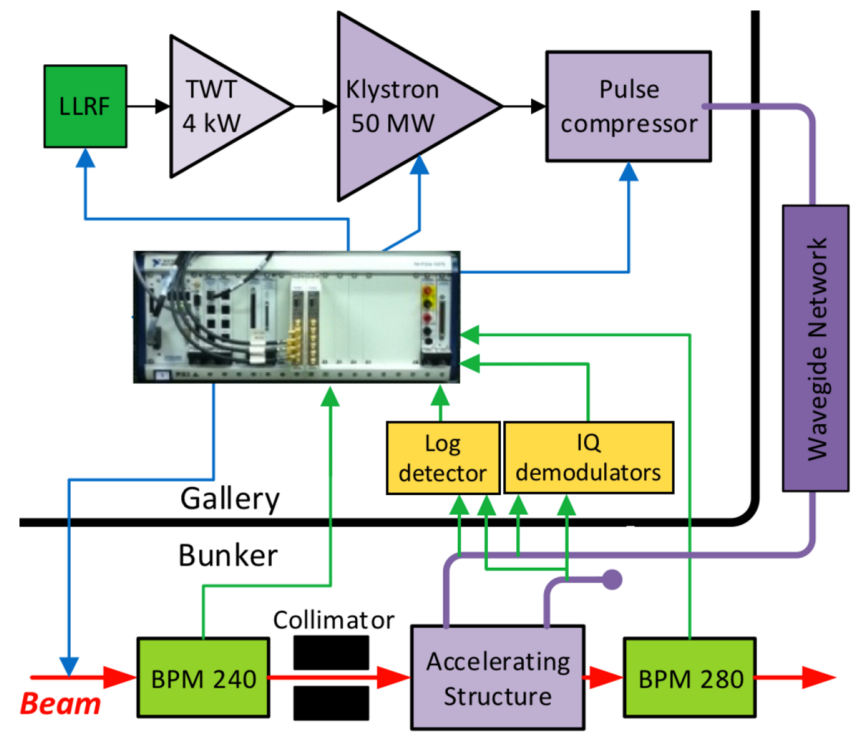

FIG. 1. Simplified layout of the hardware installation, control and acquisition system.

The acquisition system checks all signals pulse-by-pulse and stores breakdown events, along with two preceding pulses, and stores standard pulses every minute. The online trigger system results in a large number of saved events. These are then analyzed offline and typically only $~ 10 \%$ of the detected events are real breakdowns happening inside the structure under test. The online breakdown identification is based on the observation of the increase of reflected rf power and the loss of transmitted signal through the structure compared to the incident pulse [22]. This system is designed to be robust and not to discard any event of interest. The stored breakdown events use a buffer which contains the two previous pulses in addition to the breakdown pulse itself. This approach allows the breakdowns to be compared with normal events and to check for potential evidences of breakdown precursors. A more detailed description of the setup is reported in $[9,11,12]$.

\section{RESULTS}

\section{A. Conditioning and unloaded operation}

A CLIC prototype structure of type TD26CC (Tapered, Damped, 26 regular cells, compact couplers) was used to carry out the tests. It is described in [23] and some key features are outlined in Table II.

The accelerating structure was previously installed, processed and tested in another location without beam. To realise the experiment with beam it was vented, moved and installed in the CTF3 linac. The processing and performance of the tests without beam are reported in [4,24].

The structure was reconditioned in the new installation and measurements began once the structure had reached relatively stable operating gradient. This period is visible in the left part of Fig. 2, that shows the different experiments 
TABLE II. TD26CC structure parameters with CLIC main beam parameters and $100 \mathrm{MV} / \mathrm{m}$ of accelerating gradient (3 TeV CLIC baseline) [23].
Average loaded gradient

Input power

Frequency

Number of regular cells

rf phase advance per cell

Iris radius (input/output)

Group velocity (input/output)

Q factor (first/last cell)

Shunt impedance (first/last cell)

Maximum surface electric field

Structure length (including couplers)

Filling time

rf-to-beam efficiency
$100 \mathrm{MV} / \mathrm{m}$

$61.3 \mathrm{MW}$

$12 \mathrm{GHz}$

26

$2 / 3 \pi \mathrm{rad}$

$3.15 / 2.35 \mathrm{~mm}$

$1.65 / 0.83 \%$ of c

$5536 / 5738$

$81 / 103 \mathrm{M} \Omega / \mathrm{m}$

$230 \mathrm{MV} / \mathrm{m}$

$23 \mathrm{~cm}$

$67 \mathrm{~ns}$

$27.7 \%$ during the year. Even with conditioning more or less saturated some longer term variations in breakdown rate were observed. However comparisons with different conditions were made as close together in time as possible.

The rf pulse shape needed to compensate beam loading and minimize energy spread with a current of $1.2 \mathrm{~A}$ [6]. The pulse had a flat top of $180 \mathrm{~ns}$ and a rising edge which compensates the transient beam loading, as in the CLIC pulse [1]. The tests have been carried out in different loading conditions, with and without the beam with a beam current of 1.6 A. The higher current value and longer beam pulse compared to the CLIC design parameters were chosen to increase the level of beam loading and thus the effect of the beam on the BDR.

The beam presence causes a modification of the accelerating gradient profile, which is shown in Fig. 3.
When the beam is present, changing the relative rf-tobeam phase by 180 degrees makes it possible to pass from the loaded condition, when the beam is accelerated, to the antiloaded condition, when the beam is decelerated. In the latter case, the output rf power from the cavity will be increased due to the different relative phase of the rf in the cavity and the beam induced voltage. The average gradient is lower in the loaded case and is lower with increasing current. Table III summarizes the average and peak gradient values at different input power and configurations used for the experiments. The CLIC $3 \mathrm{TeV}$ nominal conditions are also included however it was not possible to operate the test rf system at the nominal $3 \mathrm{TeV}$ input power of $61.3 \mathrm{MW}$ due to limitations mainly in the waveguide network. The highest input power was 43.3 MW which gave an average unloaded accelerating gradient of $100 \mathrm{MV} / \mathrm{m}$. This is above the $72 \mathrm{MV} / \mathrm{m}$ loaded gradient of the initial energy stage of CLIC [14].

\section{B. Beam effect on the breakdown rate}

During one year of operation, the structure underwent more than 800 million rf pulses. The statistics used for the analysis described later in this work is constituted by more than 313 million rf pulses, collected using alternatively different input power levels and switching frequently between operation with and without beam. The effect of the beam on the breakdown rate has been considered in three runs at the same input power. During these timespans, the input power level was maintained at a constant level, while the operation was interleaving approximately three days with beam and four days of unloaded operation per week. The experiment schedule is shown in Fig. 2 together with the gradient and the cumulative number of breakdowns. Only the time periods related to results presented in

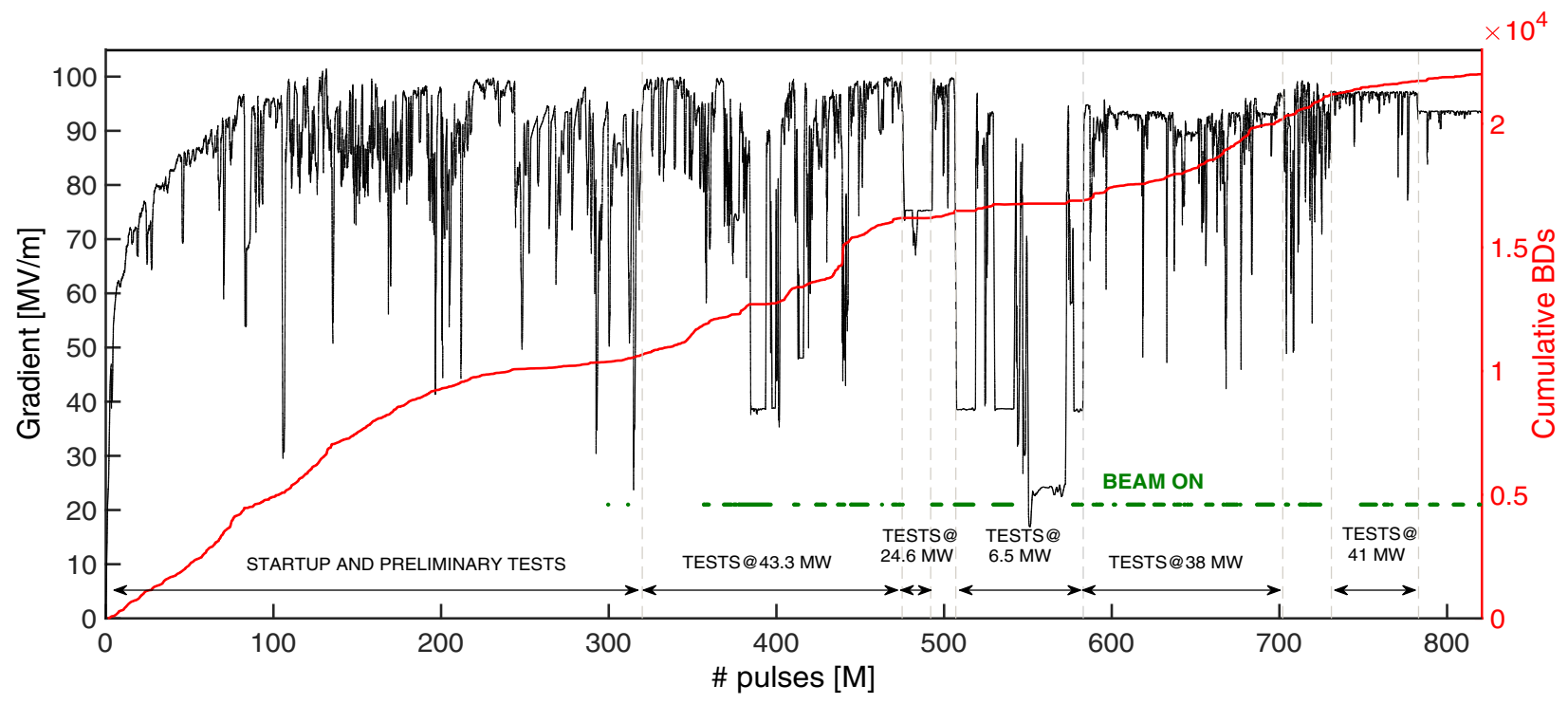

FIG. 2. Gradient and cumulative number of breakdowns during the operation of the cavity under test. The duration of tests at different input power is outlined at the bottom of the figure, and the beam presence is indicated by the green line. 


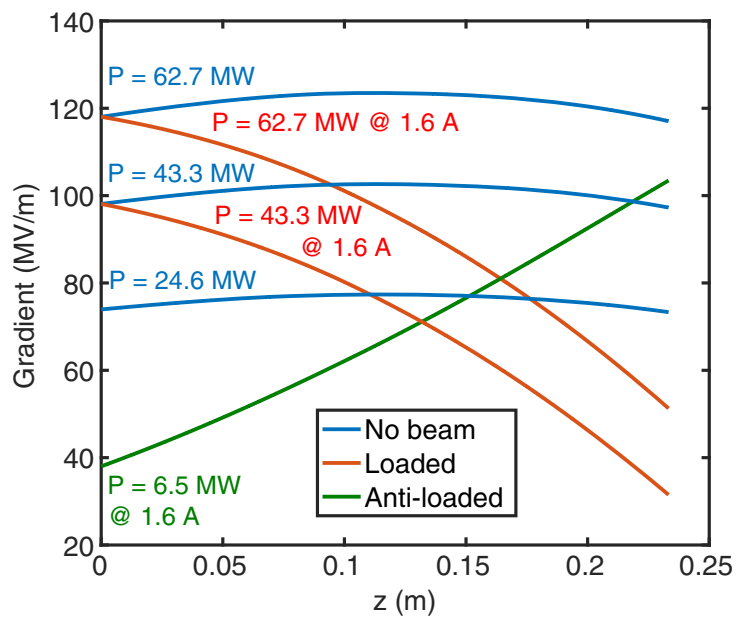

FIG. 3. Longitudinal accelerating profile for the structure under test: unloaded (blue lines) at 62.7, 43.3, and 24.6 MW input power, loaded (red lines) in the $3 \mathrm{TeV}$ CLIC design conditions for with 1.6 A of beam current at $43.3 \mathrm{MW}$ of input power, and antiloaded (green line) with the same current and 6.5 MW of input power. The gradient profile from beam $=$ loading without $\mathrm{rf}$ is given by the difference of the blue and red curve at the same input power.

this paper are marked. In case of periods of unstable operation, such as the tests at $43.3 \mathrm{MW}$, only smaller subperiods of time featuring constant gradient operation were taken into account. The measured BDRs are presented in Fig. 4. The choice of performing experiments with a breakdown rate of the order of $10^{-5}$ pulse $^{-1}$ was dictated by the time constraints.

The measurements show that, using the same input power, the breakdown rate is up to a factor 10 lower when the beam is present inside the cavity. This is expected since the breakdown probability depends on the surface field (and so on the local gradient). The lower peak gradient in the loaded case with the scaling of Eq. (1) would result in a
TABLE III. Gradient parameters in the different testing conditions and the CLIC $3 \mathrm{TeV}$ scenario. It is important to note that the tests were conducted at lower power than the $3 \mathrm{TeV}$ CLIC conditions.

\begin{tabular}{lcc}
\hline \hline Input power & $\begin{array}{c}\text { Pk. (Avg.) } \\
\text { gradient [MV/m] }\end{array}$ & Note \\
\hline 62.7 MW @ 1.18 A & $118(100)$ & $\begin{array}{c}\text { Nominal CLIC } \\
3 \mathrm{TeV} \text { scenario } \\
\text { Maximum power } \\
\text { level reached }\end{array}$ \\
43.3 MW no beam & $103(101)$ & \\
43.3 MW @ 1.6 A & $98(72)$ & Antiloading tests \\
24.6 MW no beam & $77(76)$ & $103(68)$ \\
$6.5 \mathrm{MW} \mathrm{@} \mathrm{1.6} \mathrm{A}$ &
\end{tabular}

BDR a factor 3.9 lower than the unloaded case at the same input power. The difference with this scaling can be addressed to the reduced contribution of the regions of the structure with lower gradient during the operation with beam.

The data show a correlation between the BDR and the peak gradient inside the accelerating structure, rather than the average gradient [9]. In fact the BDR of experiments with and without beam at the same average gradient is very different, while it is consistent with comparing experiments at the same peak gradient. An experiment without beam at 24.6 MW of input power was conducted in order to compare it with the case of $43.3 \mathrm{MW}$ of input power with beam. The average gradient for this lower input power is higher than for the 43.3 MW unloaded case (see Table III). The result of the run at $24.6 \mathrm{MW}$ was that after 12 million pulses no breakdown occurred, which puts an upper limit of the BDR at such power to less than $8 \times 10^{-8}$ pulse $^{-1}$, which is inconsistent corresponding to the average gradient scaling in the case with beam.

It has to be stressed that there was a large fluctuation of the BDR both for loaded and unloaded runs. These
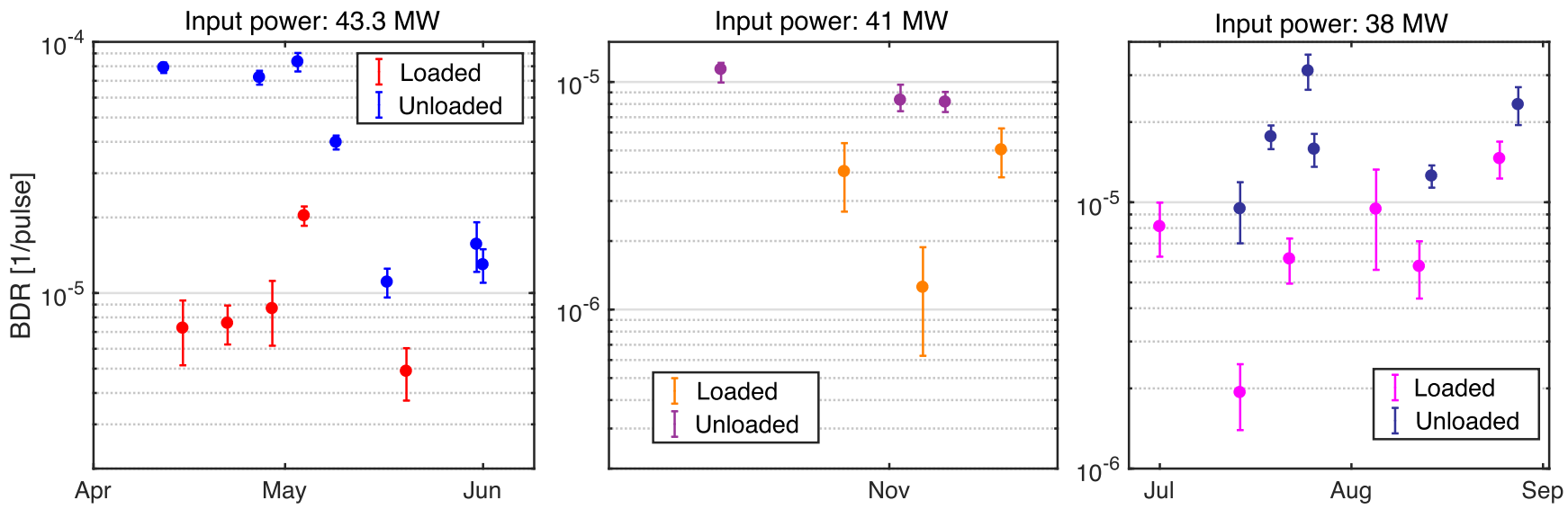

FIG. 4. Measured BDR with alternating beam presence at different input power: $43.3 \mathrm{MW}$ (left), $41 \mathrm{MW}$ (center) and $38 \mathrm{MW}$ (right). The run presenting the minimum number of breakdowns has recorded four, and normally tens of breakdowns are recorded per experiment. The error bars represent the statistical error. 
fluctuations might appear larger because the constant conditions runs were relatively short. Long term variations in BDR due to successive experiments at different input power were observed. Each test period at the same input power had interleaved tests with and without beam in order to allow the comparison of the performance.

\section{Breakdown position}

If the breakdown probability depends on the surface electric field, then the breakdown distribution along the structure will follow the trend of the longitudinal gradient profile. This implies that a region with a higher gradient is more likely to breakdown. In this experiment, considering the gradient profile in Fig. 3, the distribution of the breakdowns along the structure is expected to be relatively flat in the unloaded case; peaked in the front of the structure in the loaded case and peaked at the end of the structure in the antiloaded case.

The breakdown position can be determined from the rf power signals from the cavity. This is because the breakdown plasma acts as a short circuit, determining the fall of the transmitted power and the reflection of the incoming power after the breakdown. A review of the possible position determining algorithms can be found in [22]. The breakdown positioning algorithm used in this work compares the relative delay between the falling edge of the transmitted power and the rising edge of the reflected power to estimate the position. The edges of the signals are detected by looking at the difference with respect to the previous pulse (see Fig. 5). The location of the breakdown is later calculated using the group velocity profile.

The measured breakdown distribution for the different beam loading cases is plotted in Fig. 6 .

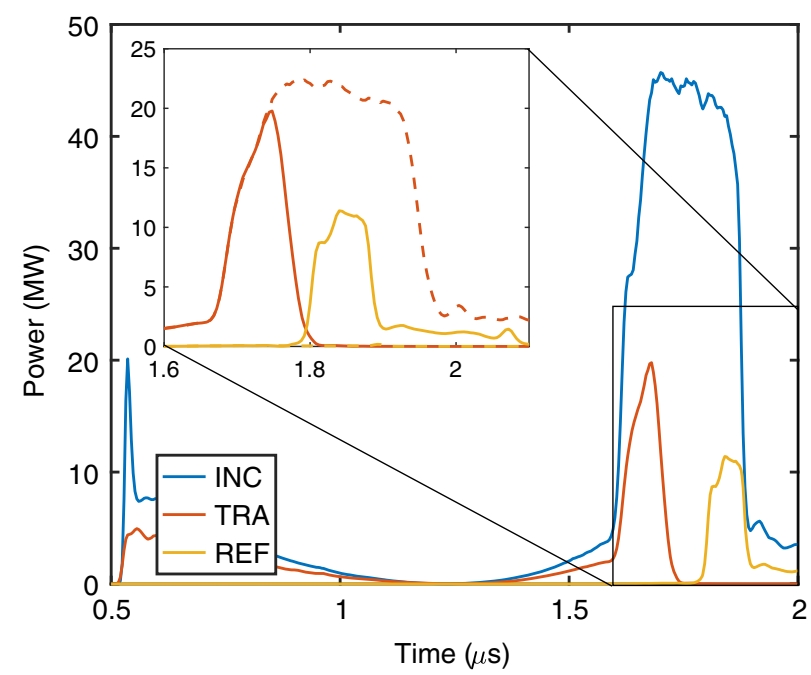

FIG. 5. rf power signals in the case of a breakdown without beam. Incident (INC), transmitted (TRA) and reflected (REF) power signals are reported. The previous pulse TRA and REF signals (with no breakdown) are reported dashed in the inset.

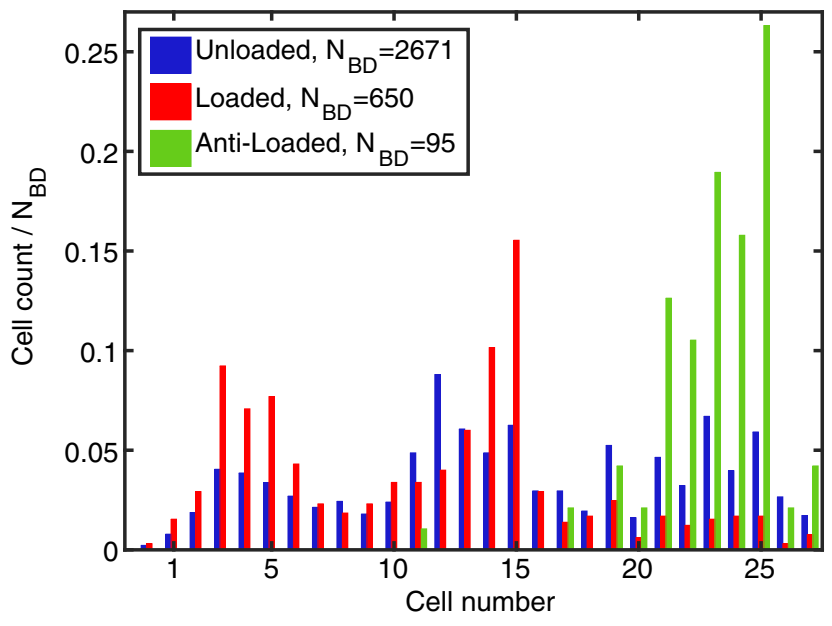

FIG. 6. Breakdown distribution in the unloaded (blue), loaded (red), and antiloaded (green). First and last bars refer to the two coupling cells.

The central part of the structure showed an unexpected higher number of BDs for both unloaded and loaded case. The origin of this anomaly is not known. It appeared after a long period of antiloaded operation. This anomaly makes it impossible to deduce the precise scaling law for the longitudinal distribution of the BDs.

The breakdown distribution in the three cases is nevertheless compatible with the reasoning given at the beginning of Sec. III C, showing a different breakdown distribution according to the gradient profile.

To deduce the scaling law ruling the longitudinal distribution of the breakdowns, repeating the experiment involving a new cavity unbiased by the more active zone in the center of the structure would be necessary.

\section{Beam effect on the rf power after the BD}

A particularity of the operation with beam is the production of $\mathrm{rf}$ power by the beam downstream of a breakdown. The rf power signals for a BD with beam where this can be seen are shown in Fig. 7. The breakdown causes a reflection of the rf power, and consequently a drop of the transmitted power. When the beam is present after, a spike in the transmitted power occurs after the breakdown. If the beam pulse has a sufficient length afterwards, a constant power level establishes up to the end of the beam pulse.

The rf production after the $\mathrm{BD}$ can be explained with beam generated rf downstream the BD location. While all the rf generated by the beam upstream the BD location is reflected there by the breakdown, the beam induced rf builds up downstream the BD location.

This is caused by the combination of the high beam current and the high level of beam loading of the structure. Since this is an effect determined purely by the beam, the plateau power never exceeds the power level induced by pure beam loading. 


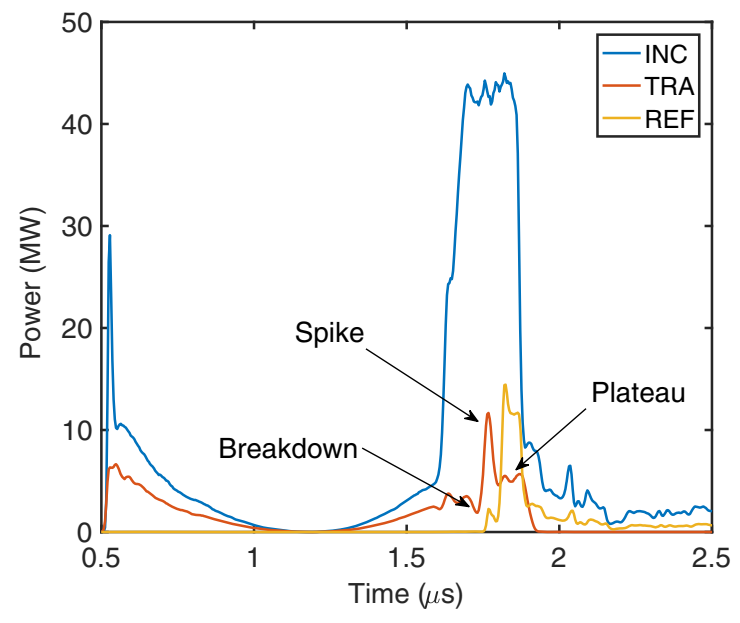

FIG. 7. rf power signals in the case of a breakdown with beam. The same color coding of Fig. 5 is used. Fast power spikes in the signals, like the spike at $0.6 \mu \mathrm{s}$ in the blue trace are artifacts of the DAQ system. The consistency of the spikes in the transmitted power, object of this section, have been validated using a parallel detector system based on diodes.

The presence of the power spike signal has been recorded with both the logarithmic and the I/Q detectors. Also the spike can not originate from higher harmonics of the klystron, as the accelerating structure acts as a filter, and none of the higher harmonics of the klystron is in the passbands of the structure.

The power spike occurs when the plasma induces a transient 180 degree phase shift which momentarily shifts the incoming rf to the decelerating, and thus rf producing, phase. The calculations show that the spike amplitude is consistent with the case without beam adding the beam loading. Such calculations confirm that the spike is due to the interaction between the beam-induced fields and the rf during the transmitted power fall time after the breakdown. This hypothesis is also supported by the fact that spikes higher than the level achievable by simple beam loading have been recorded.

Figure 8 shows a schematic representation of the effect of a breakdown occurring $10 \mathrm{~cm}$ after the entrance of the structure. The blue line represents the loaded profile during normal operation without breakdown. When the breakdown happens, the two extreme possible situations are: (1) The incoming rf is completely reflected back (bottom green line case), so the output power generated by the structure originates from the beam induced voltage only. This mechanism explains the power production regime that originates the "plateau" of Fig. 7. (2) The incoming rf power is entirely transmitted, but with a phase shift of $\pi$ (top red line case), determining a power production in the antiloading regime, as outlined in Sec. III A. These scenarios assume the steady state of the beam loading in the cavity, as described in [25]. The spike cannot be addressed using this simple model since it is a fast and dynamic perturbation, and can be induced by a number of causes.

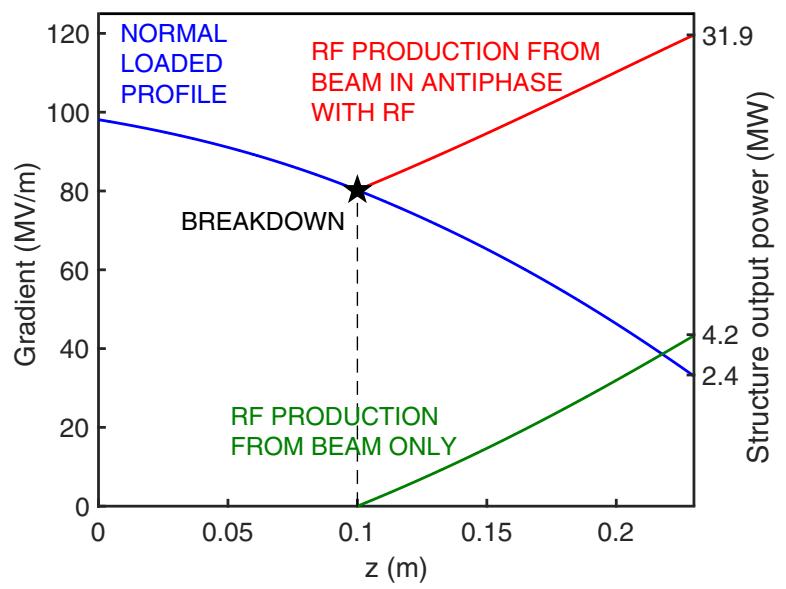

FIG. 8. Possible scenarios of $\mathrm{rf}$ power production after a breakdown at $10 \mathrm{~cm}$ after the entrance of the structure. The maximum rf production (red curve) is calculated adding the beam contribution to the unloaded profile starting at the breakdown location.

An excessive power level of the spike could damage a part of the structure not conditioned for such high fields (the last part of the structure is subjected to lower fields due to attenuation). Figure 9 shows the comparison of the highest spike in transmitted power recorded and a transmitted power during normal operation without beam. A beam-produced power level higher than the transmitted power in unloaded operation has never been observed. Considering that this is a fast dynamic effect and the beam current in the CLIC operation scenario is lower than in the tests, the power spike is not considered a risk during CLIC operation.

Figure 10 shows the transmitted rf phase during a breakdown event. Due to the effect of the breakdown the

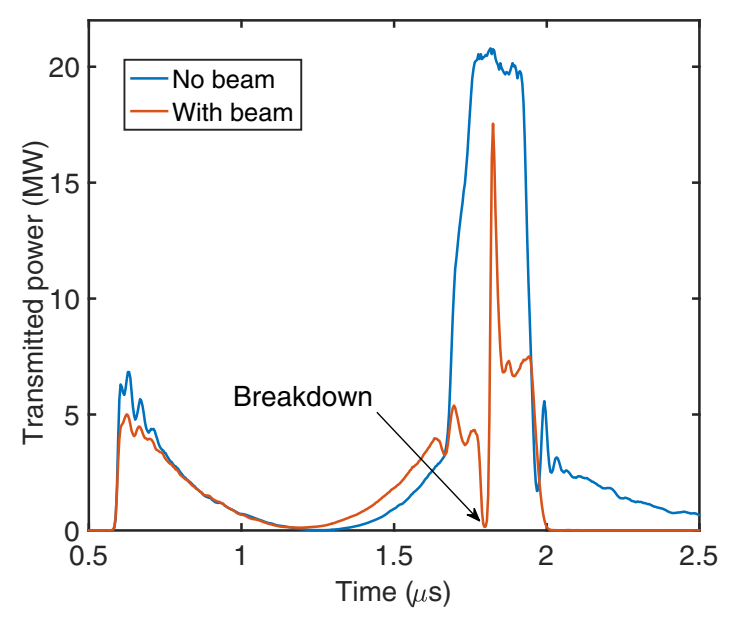

FIG. 9. Transmitted power for a reference signal during normal operation without beam (in blue), and the highest transmitted power registered in a breakdown event during operation with beam (in red). In both cases the input power is $43.3 \mathrm{MW}$. The difference in the initial part of the pulse is due to a different tuning of the pulse compressor. 


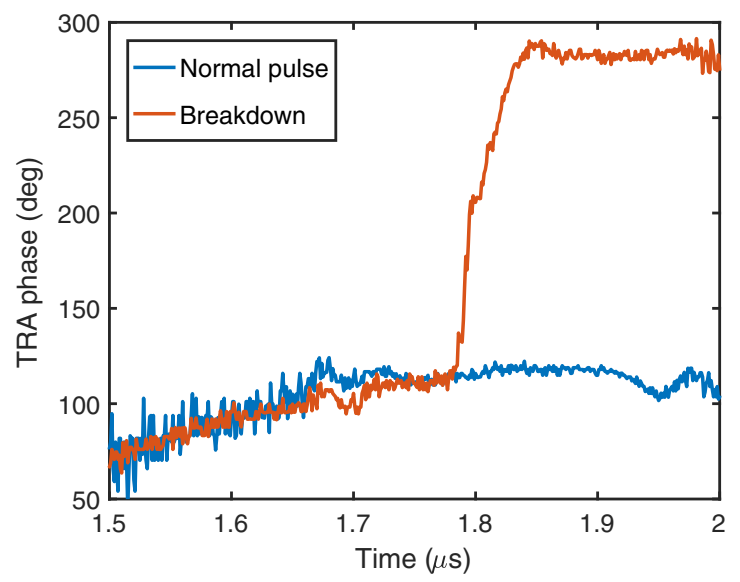

FIG. 10. Phase variation of the transmitted power signal during a breakdown with beam (red) compared to a normal rf pulse without breakdown (blue). The phase variation preceding the breakdown results from the rf pulse compression used for the rf pulse generation.

phase jumps, reaching a constant value at the beamproduced power plateau. The phase measurement before the breakdown is dominated by the incoming rf phase. After the breakdown, the incoming of is reflected, therefore the measured phase is just the beam-induced rf phase. The phase jump amplitude is constant, and corresponds to the relative phase between $\mathrm{rf}$ and beam of 180 degrees. The measured phase jump in this experiment is $190 \pm 30$ degrees, as expected for operation in oncrest acceleration.

\section{CONCLUSIONS}

The beam effect on the breakdown rate has been measured in a prototype CLIC accelerating structure (TD26CC). These measurements go beyond previous experiments, with much higher statistics and precision. A breakdown rate reduction by the beam loading up to an order of magnitude has been measured. Furthermore the effect of antiloading conditions has been studied.

Taken together these results indicate that the local BDR is determined by the local field and that the reduction of the BDR is caused by the modication of the gradient profile induced by the beam. Additional support to the local gradient effect is given by the distribution of the breakdowns inside the structure. More data have to be collected to deduce the correct scaling law for the breakdown longitudinal distribution.

Overall these results suggest that an increased structure tapering could result in a structure with a lower overall breakdown rate while operating with beam, although this would enhance breakdown rate in the structure without beam, or with lowered beam current. The rf production by the beam downstream of a $\mathrm{BD}$ has been observed but does not constitute an issue for the CLIC application. More details on setup, analysis and operation can be found in $[26,27]$.

\section{ACKNOWLEDGMENTS}

The authors would like to thank the CERN CTF and PS operators for their invaluable help with beam setup and supervision. Funding for the CLIC accelerating structure was provided by the Swiss National Science Foundation Grant No. 135012. The authors would like to thank all the past and present CERN colleagues in the CTF3, Xbox and structure fabrication teams. In particular thanks to N. Catalan-Lasheras, R. Corsini, D. Gamba, J. Giner-Navarro, A. Grudiev, G. McMonagle, L. Navarro, I. Syratchev, R. Rajamaki, X. Stragier, L. Timeo and B. Woolley.

[1] M. Aicheler, P. Burrows, M. Draper, T. Garvey, P. Lebrun, K. Peach, N. Phinney, H. Schmickler, D. Schulte, and N. Toge, A multi-TeV linear collider based on CLIC technology: CLIC conceptual design report, CERN Technical Report No. CERN-2012-007, 2012.

[2] A. Palaia, M. Jacewicz, T. Muranaka, R. Ruber, V. Ziemann, and W. Farabolini, in Proceedings of the 3rd International Particle Accelerator Conference, New Orleans, LA, 2012 (IEEE, Piscataway, 2012), p. 73.

[3] W. Wuensch, in Proceedings of International Particle Accelerator Conference (IPAC'17), Copenhagen, Denmark, 2017 (JACoW, Geneva, Switzerland, 2017), p. 24, DOI: 10.18429/JACoW-IPAC2017-MOYCA1.

[4] A. Degiovanni, S. Doebert, W. Farabolini, A. Grudiev, J. Kovermann, E. Montessinos, G. Riddone, I. Syratchev, R. Wegner, W. Wuensch, A. Solodko, and B. Woolley, in 5th International Particle Accelerator Conference (IPAC 2014), Dresden, Germany, 2014 (JACoW, Geneva, Switzerland, 2014), p. 2285, DOI: 10.18429/JACoWIPAC2014-WEPME015; Report No. CERN-ACC-20140147.

[5] P. B. Wilson, in Proceedings of 9th International Conference on High-Energy Accelerators, SLAC, Stanford, CA, USA, 1974 (1974), pp. 57-62.

[6] O. Kononenko and A. Grudiev, Transient beam-loading model and compensation in Compact Linear Collider main linac, Phys. Rev. ST Accel. Beams 14, 111001 (2011).

[7] A. Grudiev, S. Calatroni, and W. Wuensch, New local field quantity describing the high gradient limit of accelerating structures, Phys. Rev. ST Accel. Beams 12, 102001 (2009).

[8] W. Wuensch, Status and objectives of the clic x-band activity, HG2012 ICFA mini workshop (2012), http:// indico.cern.ch/event/165513.

[9] F. Tecker et al., in Proceedings, 7th International Particle Accelerator Conference (IPAC 2016): Busan, Korea, 2016 (JACoW, Geneva, Switzerland, 2016), p. THPOR031, DOI: 10.18429/JACoW-IPAC2016-THPOR031.

[10] G. Geschonke and A. Ghigo, CTF3 design report, CERN Technical Report No. CERN-PS-2002-008-RF, No. CTF3NOTE-2002-047, No. LNF-2002-008-IR, 2002.

[11] F. Tecker et al., in Proceedings of the 4th International Particle Accelerator Conference, IPAC-2013, Shanghai, China, 2013 (JACoW, Shanghai, 2013), p. TUPME054. 
[12] J. L. Navarro Quirante et al., in Proceedings of LINAC conference 2014, CERN, Geneva, Switzerland, 2014 (2014), p. TUPP033, https://accelconf.web.cern.ch/AccelConf/ LINAC2014/papers/tupp033.pdf.

[13] R. Corsini, in Proceedings of the 3rd International Particle Accelerator Conference, New Orleans, LA, 2012 (IEEE, Piscataway, 2012), p. 1101.

[14] M. J. Boland et al. (CLIC and CLICdp Collaborations), Updated baseline for a staged Compact Linear Collider, CERN Technical Report No. CERN-2016-004, 2016.

[15] J. Kovermann, N. Catalan-Lasheras, S. Curt, S. Doebert, M. Naon, G. McMonagle, E. Paju, S. Rey, G. Riddone, K. Schirm, I. Syratchev, L. Timeo, W. Wuensch, A. Hamdi, F. Peauger, J. Eichner, A. Haase, and D. Sprehn, Commissioning of the First Klystron-based X-band Power Source at CERN, Proceedings of IPAC2012 (2012), p. THPPC060, https://accelconf.web.cern.ch/Accelconf/ IPAC2012/html/auth3437.htm.

[16] B. J. Woolley, High power X-band RF test stand development and high power testing of the CLIC crab cavity, Ph.D. thesis, Lancaster University, 2015.

[17] D. Sprehn et al., A $12 \mathrm{GHz} 50 \mathrm{MW}$ klystron for support of accelerator research, Technical Report No. SLAC-PUB14377, 2011.

[18] http://www.scandinovasystems.com/.

[19] A. Fiebig and C. Schieblich, in Proceedings of EPAC90 conference, Nice, France, 1990, CERN Report No. CERNPS-90-13-RF, p. 937, https://accelconf.web.cern.ch/ AccelConf/e90/PDF/EPAC1990_0937.PDF.
[20] A. Bogdashov, G. Denisov, S. Kuzikov, A. Vikharev, K. Schirm, and I. Syratchev, in Proceedings of RuPAC2010, Protvino, Russia, 2010 (2010), p. TUPSA005, https://accelconf.web.cern.ch/AccelConf/r10/papers/ tupsa005.pdf.

[21] http://ni.com.

[22] A. Degiovanni et al., in Proceedings of LINAC conference 2014, CERN, Geneva, Switzerland, 2014 (2014), p. TUPP029, https://accelconf.web.cern.ch/AccelConf/ LINAC2014/papers/tupp029.pdf.

[23] A. Grudiev and W. Wuensch, in Proceedings of the 25th International Linear Accelerator Conference, LINAC-2010, Tsukuba, Japan (KEK, Tsukuba, 2010), p. MOP068.

[24] A. Degiovanni, W. Wuensch, and J. G. Navarro, Comparison of the conditioning of high gradient accelerating structures, Phys. Rev. Accel. Beams 19, 032001 (2016).

[25] A. Lunin, V. Yakovlev, and A. Grudiev, Analytical solutions for transient and steady state beam loading in arbitrary traveling wave accelerating structures, Phys. Rev. ST Accel. Beams 14, 052001 (2011).

[26] E. Senes, Test of the beam effect on vacuum arc occurrence in a high-gradient accelerating structure for the CLIC project, Master thesis, Phys. Dep., University of Torino, https:/github.com/esenes/Master-thesis/blob/master/thesis .pdf.

[27] J. Giner Navarro, Breakdown studies for high gradient rf warm technology in: CLIC and hadron therapy linacs, Ph.D. thesis, University of Valencia, 2016. 